\title{
Anterior Insula Integrates Information about Salience into Perceptual Decisions about Pain
}

\author{
Katja Wiech, ${ }^{1,2,3}$ Chia-shu Lin, ${ }^{2}$ Kay H. Brodersen, ${ }^{2}$ Ulrike Bingel, $, 2,4$ Markus Ploner, ${ }^{2,5}$ and Irene Tracey ${ }^{1,2}$ \\ ${ }^{1}$ Nuffield Department of Anaesthetics and ${ }^{2}$ Oxford Centre for Functional Magnetic Resonance Imaging of the Brain, Department of Clinical Neurology, \\ University of Oxford, John Radcliffe Hospital, Oxford OX3 9DU, United Kingdom, ${ }^{3}$ Research Group Health Psychology, Department of Psychology, \\ University of Leuven, 3000 Leuven, Belgium, ${ }^{4}$ NeuroImage Nord, Department of Neurology, University Medical Center Hamburg-Eppendorf, 20246 \\ Germany, and ${ }^{5}$ Department of Neurology, Technische Universität München, 81675 Munich, Germany
}

The decision as to whether a sensation is perceived as painful does not only depend on sensory input but also on the significance of the stimulus. Here, we show that the degree to which an impending stimulus is interpreted as threatening biases perceptual decisions about pain and that this bias toward pain manifests before stimulus encounter. Using functional magnetic resonance imaging we investigated the neural mechanisms underlying the influence of an experimental manipulation of threat on the perception of laser stimuli as painful. In a near-threshold pain detection paradigm, physically identical stimuli were applied under the participants' assumption that the stimulation is entirely safe (low threat) or potentially harmful (high threat). As hypothesized, significantly more stimuli were rated as painful in the high threat condition. This context-dependent classification of a stimulus as painful was predicted by the prestimulus signal level in the anterior insula, suggesting that this structure integrates information about the significance of a stimulus into the decision about pain. The anticipation of pain increased the prestimulus functional connectivity between the anterior insula and the midcingulate cortex (MCC), a region that was significantly more active during stimulation the more a participant was biased to rate the stimulation as painful under high threat. These findings provide evidence that the anterior insula and MCC as a "salience network" integrate information about the significance of an impending stimulation into perceptual decision-making in the context of pain.

\section{Introduction}

Pain indicates that action is required to prevent or minimize further harm to the organism. Hence the classification of a sensation as painful is critical to ensure that protective responses can be initiated in time. Functional neuroimaging studies have begun to investigate the neural mechanisms underlying perceptual decisions in the visual (Heekeren et al., 2004), auditory (Binder et al., 2004; Kaiser et al., 2007) and somatosensory domain (Pleger et al., 2006; Preuschhof et al., 2006). In contrast, very little is known regarding the perceptual decision of a stimulus as painful. Existing studies on perceptual decisions have focused on the integration of sensory evidence and the formation of a decision variable following stimulus processing. There is, however, increasing evidence suggesting that a percept is critically determined by the momentary configuration of relevant brain areas before stimulus encounter (Arieli et al., 1996; Supèr et al., 2003; de Lafuente and Romo, 2005; Boly et al., 2007; Hesselmann et al., 2008).

In a recent functional magnetic resonance imaging (fMRI) study, we showed that the activation level of the bilateral anterior

Received April 21, 2010; revised July 2, 2010; accepted July 31, 2010.

The contribution of K.W. was supported by an Odysseus Grant by the "Fund for Scientific Research" —Flanders, Belgium (FW0). We thank Geert Crombez for his suggestions for the threat manipulation and Stuart Wilson for help with fMRI data acquisition. We thank the Medical Research Council of Great Britain and Northern Ireland (FMRIB (entre).

Correspondence should be addressed to Dr. Katja Wiech, Oxford Centre for Functional Magnetic Resonance Imaging of the Brain (FMRIB), John Raddliffe Hospital, Headington, 0X3 9DU, UK. E-mail: kwiech@fmrib.ox.ac.uk. DOI:10.1523/JNEUROSCI.2087-10.2010

Copyright $\odot 2010$ the authors $\quad 0270-6474 / 10 / 3016324-08 \$ 15.00 / 0$ insula predicted whether a laser stimulus calibrated at individual pain threshold intensity is perceived as painful or not (Ploner et al., 2010). The more active the anterior insula before stimulus application, the more likely the subsequently applied stimulus was rated as painful. Furthermore, the prestimulus functional connectivity between the anterior insula and pain-modulatory brain regions [e.g., periaqueductal gray (PAG)] differed between physically identical trials that were rated as painful and trials perceived as nonpainful. These influences of the anterior insula on perception were observed without any manipulation of the behavioral context.

Adaptive behavior includes seeking information about future events to predict and control them. This applies particularly to experiences which signal potential harm such as pain. The prestimulus state of brain areas involved in perceptual decisions may therefore also reflect information about the significance of an impending stimulus. For instance, Sarinopoulos et al. (2010) recently showed that uncertainty-related expectations lead to an overestimation of the frequency by which uncertain cues are followed by negative outcome and amplified neural responses to aversion. Importantly, it is the subjective interpretation of available information that influences perceptions and actions, regardless of whether it is factually true or not. For instance, the interpretation of an event as threatening has been shown to increase stimulus salience and to amplify the perception of pain and nonpainful stimuli (Smith et al., 1998; Sawamoto et al., 2000; Severeijns et al., 2002; de Gier et al., 2003; Arntz and Claassens, 2004; Moseley and Arntz, 2007; Vlaeyen et al., 2009) and related 
threat manipulation

high threat low threat

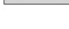

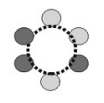

high threat: "application of
laser stimulis is approved with reservations"

low threat: "application of laser stimulis is fully approved"

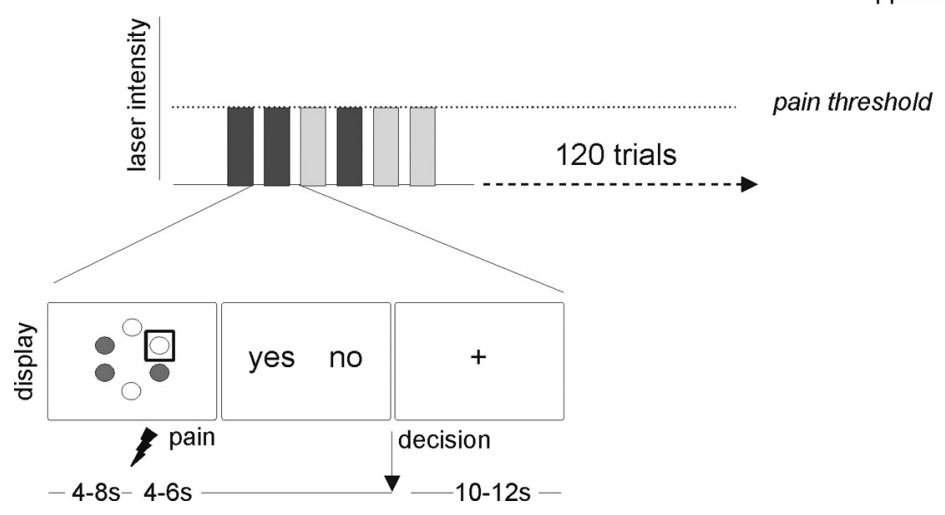

Figure 1. Experimental design. A graphic representation of the six stimulation sites was shown before stimulus application. Fully approved sites were shown in a different color than sites approved with reservations. The site stimulated in the current trial was highlighted by a gray square. Subsequently, the laser stimulus calibrated to pain-threshold intensity was applied to the right foot. Participants were then prompted to indicate by a button press (here, left button for "yes" = pain and right button for " $\mathrm{no"}=$ no pain) whether the stimulus had been perceived as painful or not. Assignment of buttons was varied from trial to trial. Following the button press, the presentation of a fixation cross ended the trial.

cardiovascular responses (Paine et al., 2009). An increased perception of threat has therefore been implicated in different processes associated with an amplified pain perception, including attention to pain (Eccleston and Crombez, 1999), anxiety or fear (Asmundson and Katz, 2009), and catastrophizing (Jackson et al., 2005). However, the role of perceived threat and salience in perceptual decisions of pain and their neural underpinnings are still unclear.

Using fMRI, we investigated the neural mechanisms underlying the effect of perceived threat and increased stimulus salience on perceptual decisions of pain by combining a previously established pain-detection paradigm (Ploner et al., 2010) with a manipulation of threat. We hypothesize that a higher threat value of an impending near-pain threshold stimulation induced by the information participants receive will bias the perception of these stimuli toward pain. Moreover, we hypothesize that the anterior insula integrates information about the threat value of the stimulation before stimulus encounter and that this would be reflected by its engagement during the anticipation period and in its functional connectivity to brain areas involved in generating a pain perception.

\section{Materials and Methods}

\section{Subjects}

Sixteen healthy volunteers participated in the study ( 11 females; mean age, 24; range, 19-30). Participants were right-handed, displayed normal pain thresholds at the site of stimulus application and had no history of neurological or psychiatric disease or chronic pain. All subjects participated having given full informed consent, and the study was approved by the local research ethics committee.

\section{Experimental paradigm}

Using fMRI, we investigated brain responses during the anticipation and perception of physically identical laser stimuli perceived as painful or nonpainful under low and high threat instruction $(2 \times 2$ factorial design). The experiment was divided into four runs each consisting of 30 trials. On each trial, a laser stimulus calibrated at the individual painthreshold level was applied to the dorsum of the right foot. Before stim- ulus application, subjects were informed whether the stimulus would be delivered to a "low threat" or a "high threat" site (15 stimuli applied to a low threat site, 15 stimuli applied to a high threat site per run; see Threat manipulation). The order of low and high threat trials was pseudo-randomized with no more than two consecutive trials of the same type. Following stimulus delivery, participants were prompted to indicate whether this stimulus had been perceived as painful or not. Depending on this decision each trial was classified as a pain or a no pain trial. The near-threshold stimulation paradigm had been used in a recent study by our group (Ploner et al., 2010).

\section{Threat manipulation}

To manipulate the perceived threat value of the stimulation all participants underwent an experimental manipulation of the threat value of the laser stimulation before they were positioned in the scanner. In this manipulation, a red light was positioned over six predefined sites on the dorsum of the right foot that were later on stimulated during the experiment. Participants were told that the purpose of this procedure was to assess the properties of the skin on the stimulation sites to ensure that the application of the laser stimuli is safe. Depending on the properties of the skin, each stimulation site would be classified as (1) fully approved, indicating that the skin seemed robust enough to tolerate laser stimulation (low threat condition), (2) approved with reservations, that is the site of stimulation would have to be closely monitored by the participant during the experiment to detect potential changes in perception to prevent skin damage (high threat condition), or (3) not approved because laser stimulation might cause serious harm to the tissue. Each of the sites was marked by a color-coded circle (not filled) on the skin according to the outcome of this threat manipulation. Unknown to subjects, the device used for "skin assessment" emitted a beam of light onto the skin but did not allow for testing of any skin properties. It was predefined that three of the six test sites would be classified as fully approved and three sites would be approved with reservations. The aim of this design was to ensure that the stimulation would be perceived as more threatening in half of the trials and as less threatening in the other half. The assignment of the six stimulation sites to the two outcomes (i.e., fully approved vs approved with reservations) and the color-coding were randomized between subjects.

\section{Experimental procedures}

During the fMRI experiment participants were shown a schematic representation of the color-coded six sites of stimulation (Fig. 1) before the application of each laser stimulus. A gray square, positioned around one of the sites, informed them which site would be stimulated next. These cues were presented on a projection screen that was positioned at the feet of the participants and remained on the screen until the end of the laser stimulation. Four to $8 \mathrm{~s}$ after the onset of the cue ("cue period"), the laser stimulus was delivered to the cued site ("stimulation period"). Four to $6 \mathrm{~s}$ after stimulus delivery, subjects were visually prompted to indicate their decision by pressing one of the two response buttons without any time constraints. The allocation of buttons to indicate the perception of pain ("yes") or no pain ("no") was fully counterbalanced within and between subjects. Each trial was concluded by a variable intertrial interval (ITI) of $10-12$ s during which a fixation cross was shown. At the end of each of the four runs, subjects were prompted to rate the average level of pain intensity and anxiety during both high threat and low threat trials on a Visual Analog Scale (VAS) with the anchor points " $0=$ not painful/anxious at all" to " $100=$ very painful/anxious" (duration: 10 s per rating). Note that pain intensity ratings only refer to trials that were perceived as painful. Stimulus presentation and response recording were implemented using Presentation (Neurobehavioral Systems). 


\section{Laser stimulation}

Cutaneous heat stimulation was delivered by a Nd:YAP laser (DEKA) with a wavelength of $1340 \mathrm{~nm}$, pulse duration of $4 \mathrm{~ms}$ and spot diameter of $5 \mathrm{~mm}$. At painful intensities these stimuli yield a pinprick-like sensation, whereas at nonpainful intensities stimuli are perceived as warm or not perceived at all. To avoid sensitization or fatigue of primary nociceptive afferents, the laser beam was moved to a different position after each stimulus. After participants had been positioned in the magnetic resonance scanner, pain thresholds were determined using a modified methods of limits approach (Warnaby, 2005). To ensure that the stimulation intensity was as close as possible to the individual pain threshold throughout the experiment the same calibration procedure was repeated before each of the four runs. The average laser intensity at pain threshold level was $1.44 \mathrm{~J}$ (SD, 0.23 ; range: $1.0-1.75 \mathrm{~J}$ ) for the first run, $1.63 \mathrm{~J}$ for the second run ( $\mathrm{SD}, 0.33$; range: $1.0-2.25 \mathrm{~J}), 1.83 \mathrm{~J}$ for the third run $(\mathrm{SD}$, 0.42 ; range: $1.25-3.0 \mathrm{~J})$ and $1.95 \mathrm{~J}$ for the fourth run $(\mathrm{SD}, 0.56$; range: $1.25-3.75 \mathrm{~J})$. Given that the same number of threat and non-threat trials was presented in each run, adjustments in physical stimulation intensity between runs applied to both trial types.

\section{Analysis of behavioral data}

First, we tested whether the number of trials rated as painful and the number of trials rated as nonpainful were significantly different by performing a paired $t$ test. A nonsignificant difference would confirm that overall the laser intensity was successfully calibrated at pain-threshold level. Subsequently, the choice behavior and pain and anxiety ratings were compared between the high and low threat condition, again using paired $t$ tests. With regard to choice behavior, the number of trials that were perceived as painful in the two conditions was entered into the analysis. For pain intensity and anxiety ratings, mean ratings across all four runs were determined for both high and low threat for each participant.

\section{fMRI data acquisition}

Functional imaging was performed on a 3T MRI system (Oxford Magnet Technology) using a Nova Medical Inc. quadrature birdcage coil. Fortyone axial slices (slice thickness $3 \mathrm{~mm}$ ) were acquired using a gradient echo echoplanar T2*-sensitive sequence (repetition time, $3 \mathrm{~s}$, echo time, $30 \mathrm{~ms}$; flip angle, $90^{\circ}$; matrix, $64 \times 64$; field of view, $192 \times 192 \mathrm{~mm}^{2}$ ). The first four images were discarded to compensate for $\mathrm{T} 1$ saturation effects.

\section{Image processing}

Image processing and statistical analyses were performed using SPM8 (http://www.fil.ion.ucl.ac.uk/spm). To correct for subject motion, images were realigned to the first volume and unwarped (Andersson et al., 2001). Images of all sessions were spatially normalized to a standard echoplanar imaging template included in the SPM software package (Friston et al., 1995) using a fourth-degree B-spline interpolation, and finally smoothed with an isotropic $8 \mathrm{~mm}$ FWHM three-dimensional Gaussian filter.

\section{fMRI statistical analysis}

To investigate the main effect of pain (i.e., "pain" vs "no pain" across high threat and low threat trials) and the main effect of threat (i.e., "high threat" vs "low threat") during anticipation and stimulation, we estimated subject-specific (first-level) linear models that included regressors for all four combinations of threat (high vs low) and pain (pain vs no pain) in both periods. Button presses were included as a regressor-of-nointerest. Anticipation periods were modeled according to their respective duration (i.e., $4-8 \mathrm{~s}$ ). The modeled stimulus duration was $1 \mathrm{~s}$. Serial autocorrelation was modeled as a first-order autoregressive model, and the data were high-pass filtered at a cutoff of $128 \mathrm{~s}$. All inferences on the anticipation and stimulation period were made at the second (betweensubject) level by entering the appropriate contrast into an ANOVA.

In a first step, main effects of both factors (i.e., threat and pain) and their interactions were calculated separately for both time periods, the anticipation and the stimulation period. The first interaction analysis tested for brain regions showing a larger activation difference between pain and no pain trials under high relative to low threat ([painno pain $\left.]_{\text {high threat }}-[\text { pain-no pain }]_{\text {low threat }}\right)$. The second interaction identified brain regions showing a increased activation difference between pain and no pain trials under low relative to high threat ([pain-no pain $]_{\text {low threat }}$-[pain-no pain $\left.]_{\text {high threat }}\right)$. For results of both interaction analyses see supplemental Data (available at www. jneurosci.org as supplemental material). To explore the neural mechanisms underlying the biasing effect of threat on pain perception we performed three more specific analyses.

Conjunction analysis. First, we tested which of the brain regions that showed increased activation during pain trials were also sensitive to our threat manipulation. To this end, we performed a conjunction analysis on the two main effects during the anticipation period (i.e., "pain $>$ no pain" and high vs low threat) that revealed significant activation in the anterior insula (see Results).

Psychophysiological interaction analysis. Second, to identify brain regions exhibiting a change in functional connectivity with the anterior insula depending on whether pain or no pain was anticipated we performed a psychophysiological interaction analysis (PPI) (Friston et al., 1997). For each individual we extracted the BOLD time-series from the voxels within a $4 \mathrm{~mm}$ sphere surrounding the activation peak of the anterior insula cluster showing a main effect of threat (high vs low threat) during anticipation (right anterior insula: $33,23,-8$ for $x, y, z$; left anterior insula: $-30,20,-5$ for $x, y, z ; 4 \mathrm{~mm}$ sphere). Next, PPI regressors were computed as the element-by-element product of the meancorrected anterior insula activity and a vector coding for the differential effect of pain and no pain. The individual contrast images reflecting the interaction between the psychological variable (i.e., anticipation of pain) and the activation time course in the anterior insula were subsequently entered into a one-sample $t$ test. To test whether the functional connectivity between the anterior insula and the PAG changed as in our previous study (Ploner et al., 2010), we performed a region-of-interest analysis for the PAG ( $x, y, z: 0,-26,-16 ; 4 \mathrm{~mm}$ sphere) in addition to the whole brain analysis.

Regression analyses. As outlined in the Results, the PPI analysis revealed an increased functional connectivity between the anterior insula and the anterior part of the midcingulate cortex (MCC). To explore the role of the MCC and the functional significance of its enhanced coupling with the anterior insula during anticipation, we performed two additional analyses as a third analysis step. First, we performed a simple regression analysis to test whether the degree of coupling between the anterior insula and the MCC during anticipation predicted the threatrelated bias toward pain. To obtain an individual measure for this bias, we calculated the ratio of pain trials in high threat divided by the number of pain trials in low threat trials for each participant. In the following, we will refer to this measure as the bias index. A bias index of 1 indicates the absence of a bias toward pain (i.e., the same number of trials were rated as painful in the high and the low threat condition), values $>1$ indicate the presence of a bias. All 16 subjects showed a threat-related bias toward pain $($ mean $=1.52, \mathrm{SD}=0.69)$. First level PPI contrasts reflecting the interaction between the psychological variable (anticipation of pain vs no pain) and the activation time course in the anterior insula were entered into second-level regression analyses using the bias index as the covariate.

Second, we investigated whether the MCC activation during stimulus application scaled with the individual threat-related bias toward pain. If the increased coupling between the anterior insula and the MCC indeed reflected a preparatory response, we would expect subsequent MCC engagement during stimulation to be more pronounced in participants with a stronger bias toward pain under high threat. The bias index was entered as a covariate into a second level simple regression analysis for the contrast of high threat compared with low threat trials during stimulation. In both regression analyses, region of interest analyses were performed for the MCC identified in the PPI analysis $(x, y, z=3,17,22 ; 4$ $\mathrm{mm}$ sphere) in addition to whole brain analyses.

As our study was designed to extend the paradigm established in our previous study (Ploner et al., 2010), results are reported using the same protocol for statistical thresholding, i.e., at a threshold of $p<0.001$ uncorrected, except for a priori hypothesized regions that were thresholded at $p<0.005$, uncorrected. A priori regions included pain-related brain regions of the primary and secondary somatosensory cortex, in- 
A Decisions

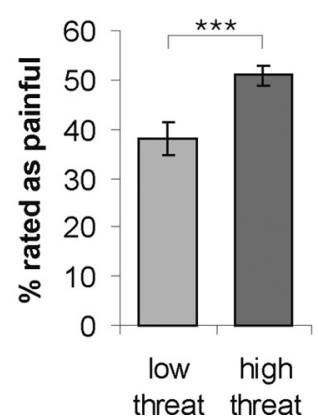

B

Pain intensity rating

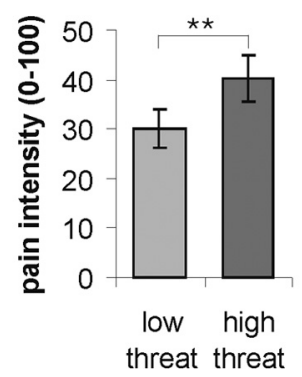

C Anxiety rating

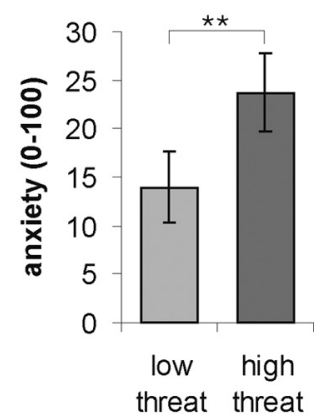

Figure 2. Behavioral data. $A$, In the high threat condition, participants perceived significantly more trials as painful compared with the low threat condition. $B, C$, At the end of the experiment, participants rated the high-threat trials as more painful relative to low-threat trials $(\boldsymbol{B})$ and felt significantly more anxious $(\boldsymbol{C})$. Error bars indicate SEs. ${ }^{* *}: p<0.001 ;{ }^{* *}: p<0.01$.

sula, anterior cingulate, orbitofrontal, medial and lateral prefrontal cortex. All reported coordinates are given in MNI space.

\section{Results}

\section{Behavioral results}

As intended by our experimental design, no significant difference was found between the number of stimuli rated as painful and those rated as nonpainful across all trials $(p>0.05)$. On average, $47 \%$ of all trials were rated as painful $(S D=10.3$; minimum: $28 \%$, maximum: $61 \%$ ). In contrast, the number of trials classified as painful was significantly higher in the high threat trials than in the low threat trials $\left(t_{(15)}=-5.74, p<0.001\right.$; Fig. $\left.2 A\right)$, indicating that our threat manipulation had been successful. Furthermore, during high threat trials, stimuli were perceived as more painful $\left(t_{(15)}=-3.73, p=0.002\right.$; Fig. $\left.2 B\right)$ and participants felt more anxious $\left(t_{(15)}=-3.25, p=0.005\right.$; Fig. $\left.2 C\right)$ than in low threat trials. This suggests that an increased perceived threat value does not only lead to a quantitative change in perception as indicated by the difference in pain intensity but also to a qualitative change from no pain to pain.

\section{fMRI results}

Pain versus no pain during anticipation and stimulation

To identify brain regions that were more active before stimuli that were subsequently classified as painful compared with nonpainful stimuli, we analyzed the time window before the application of the laser stimulus regardless of the threat condition. The anticipation period of painful compared with nonpainful trials was characterized by an increased activation in the right anterior insula confirming our previous results obtained using a similar experimental design (Ploner et al., 2010). Additional activations were found in the left insula and right amygdala (supplemental Table 1, available at www.jneurosci.org as supplemental material). The reverse contrast testing for increased activation before stimuli rated as nonpainful compared with painful, showed that no cluster survived the threshold of $p<0.001$ uncorrected (supplemental Table 2, available at www.jneurosci.org as supplemental material for threshold $p<0.005$ uncorrected).

During the application of the laser stimuli, trials classified as painful were characterized by increased activation in several brain regions commonly associated with pain processing such as the MCC, contralateral posterior insula, and orbitofrontal cortex (supplemental Table 3, available at www.jneurosci.org as supplemental material). The analysis testing for signal increase during nonpainful compared with painful trials did not yield any significant results.

High versus low threat during anticipation and stimulation

The main effect of threat (summed over painful and nonpainful trials) identified regions responding to the presentation of the visual cue signaling the pending stimulation of a site that had been labeled as "approved with reservations" compared with "fully approved" sites (high threatlow threat). This contrast revealed significant activation in the left anterior insula, SII, orbitofrontal cortex, and dorsolateral prefrontal cortex (supplemental Table 4, available at www.jneurosci.org as supplemental material). The contrast low threathigh threat revealed significant activations in bilateral dorsomedial and dorsolateral prefrontal cortex (supplemental Table 5, available at www.jneurosci. org as supplemental material).

Stimuli applied in the high-threat compared with the lowthreat condition led to increased activation in the rostral anterior cingulate cortex extending into the medial prefrontal cortex (supplemental Table 6, available at www.jneurosci.org as supplemental material). The reverse contrast testing for brain regions specifically activated during low threat compared with high threat trial revealed a small activation cluster in the temporal pole and cerebellum (supplemental Table 7, available at www.jneurosci.org as supplemental material).

\section{Brain regions integrating the level of threat into the decision about pain}

To identify brain regions showing sensitivity to both factors (i.e., pain and threat) before the application of the laser stimuli, we performed a conjunction analysis on the two main effects (i.e., pain $>$ no pain and high $>$ low threat). This analysis revealed significant activation in the anterior insula in both hemispheres only (right: $x, y, z=33,23,-8 ; z=2.81$; left: $x, y, z=-27,23$, $-2, z=2.88$; Fig. 3 ), suggesting that these brain regions integrate information about the threat value of a stimulation into the decision about pain.

\section{PPI analyses: functional connectivity of anterior insula during anticipation}

To identify brain regions showing an increase coupling with the anterior insula when pain is anticipated, we performed a PPI analysis for both the right and left anterior insula. No significant result was found for the right anterior insula. In contrast, the left anterior insula exhibited increased coupling particularly with the midcingulate cortex $(x, y, z=3,17,22)$ (Fig. 4; supplemental Table 8, available at www.jneurosci.org as supplemental material). This result indicates that when the stimulation is expected to be painful (which is more likely under high than under low threat), the coupling between the anterior insula as the key region holding this information and the MCC becomes stronger before stimulus application. The regions-of-interest analysis on the $\mathrm{PAG}$ previously reported to covary with the anterior insula (Ploner et al., 2010) did not reveal any significant result. 
A

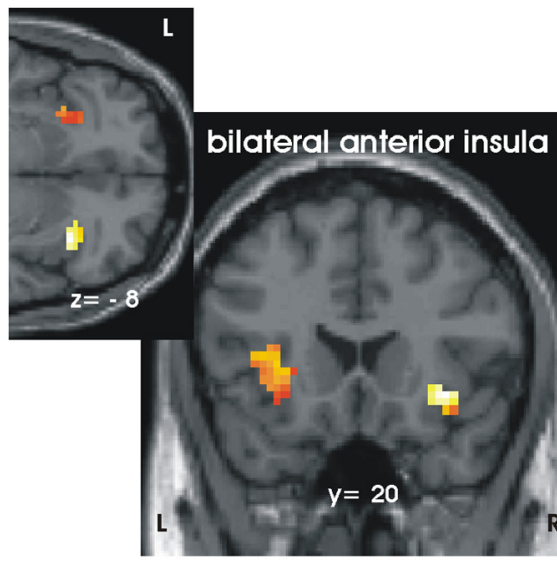

B

\begin{abstract}
left anterior insula during anticipation
\end{abstract}

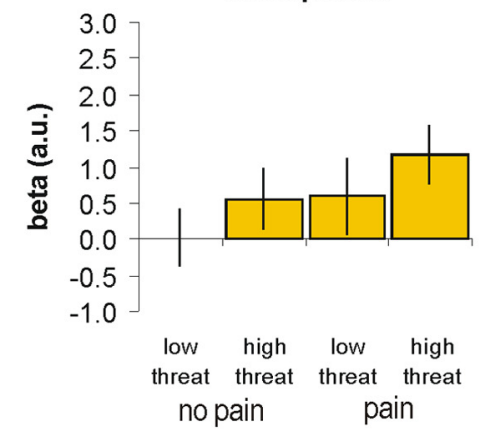

C right anterior insula during anticipation

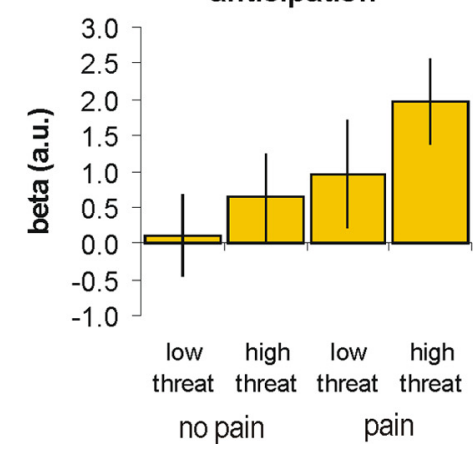

Figure 3. Anterior insula integrating information about threat-value into the decision about pain ( $L$, left; $R$, right). $A$, As revealed by a conjunction analysis, the right and left anterior insula encoded the anticipation of pain (i.e., pain $>$ no pain) and the processing of threat information (i.e., high $>$ low threat) in the anticipation period ( $p<0.005$ uncorrected). For visualization, activations are shown at a threshold of 0.05 uncorrected. $\boldsymbol{B}, \boldsymbol{C}$, Parameter estimates for the peak voxels in left $(\boldsymbol{B})$ and right anterior insula ( () plotted for illustration purpose (adjusted for effects of interest). Error bars indicate SEs.

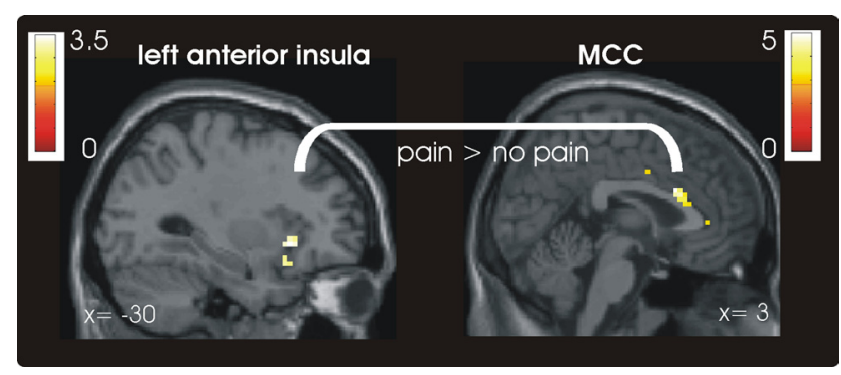

Figure 4. MCC showing a significant context-dependent covariation with the left anterior insula during anticipation.
Further analyses on the role of the MCC: correlations with individual threat-related bias toward pain

To explore the role of the MCC in more detail, we performed two additional analyses. First, we hypothesized that if the increased connectivity between the left anterior insula and the MCC during anticipation is functionally significant for the perception of the laser stimuli as painful, it should be more pronounced in participants showing a stronger threat-related bias toward pain. We therefore investigated whether the coupling between MCC and left anterior insula was predictive of the individual choice behavior. This regression analysis using a region of interest (ROI) approach did not reveal a significant result for the MCC found in the initial PPI analysis $(x, y, z=3,17,22)$. However, the whole brain analysis revealed that the coupling between the anterior insula in a slightly more lateral cluster of the MCC was positively correlated with the bias index $(x, y, z=12,14,28 ; z=2.67$; supplemental Table 9, available at www.jneurosci.org as supplemental material). In a next step, we turned to the stimulation period. If the increased coupling between left anterior insula and the MCC during anticipation reflects a prestimulus "tuning" of the MCC that becomes apparent when the stimulus is applied, participants should show an increased MCC activation during high compared with low threat stimulation the more they are biased toward pain in the high threat condition. To test this hypothesis, we investigated whether the MCC that showed increased coupling with the left anterior insula during anticipation was more activated in high compared with low threat trials during stimulation in participants with a stronger bias toward pain. The results of this regression analysis show that the bias index was indeed positively correlated with activation of the MCC during stimulation $(x, y, z=-3,11,22 ; z=5.57$, Fig. 5; supplemental Table 10, available at www.jneurosci.org as supplemental material; correlation coefficient for $4 \mathrm{~mm}$ sphere around the peak voxel: $r=0.944, p<0.001)$. As confirmed by a subsequent ROI analysis, this activation cluster in the MCC was in a similar location as the MCC exhibiting increased functional connectivity with the left anterior insula during anticipation $(x, y, z=0,11,25$; $z=4.79$; familywise error correction $p<0.05)$.

Given that 2 of the 16 subjects showed a particularly strong bias to perceive pain under high threat, we plotted the relative frequency of decisions for pain (high threat/low threat) against the activation in these brain regions for each individual to assess whether these correlations were primarily driven by the two outliers. As shown in Figure 5B, the correlation decreased upon exclusion of the two participants but remained statistically significant $(r=0.75, p<0.01)$.

\section{Discussion}

In this study, we investigated how the brain integrates the threat value of an impending stimulation into the processing of this stimulus to ensure that harmful stimuli are reliably detected. Our behavioral results show that stimuli are more likely to be rated as painful if they are believed to be potentially harmful. At the neural level, we found that the prestimulus activation in the anterior insula reflected the integration of the perceived threat value of the stimulation into the decision about pain. Furthermore, the left anterior insula showed a change in prestimulus functional connectivity with the MCC, a key region in cognitive pain modulation, as a function of pain anticipation. The same cluster in the MCC was subsequently more engaged during stimulus application the stronger the threatrelated bias toward the classification of the stimuli as painful.

Our data indicate that-regardless of the threat manipulation-prestimulus activation of the anterior insula is predictive 

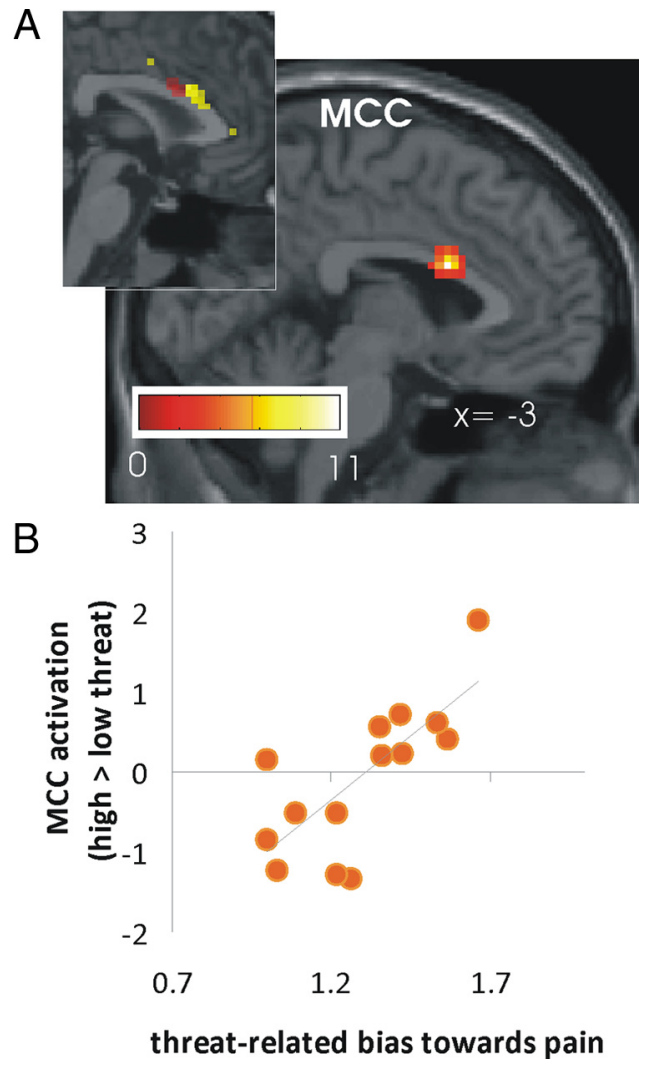

Figure 5. Second-level analysis showing a positive correlation between the threat-related bias toward pain and brain activation in high threat vs low threat trials during stimulation. $A$, The simple linear regression analysis showed a positive correlation in the MCC between the bias index used (number of pain decisions in high threat trials divided by number of pain decisions in low threat trials) and contrast maps for high $>$ low threat during stimulation. As shown in the insert this MCC activation cluster overlapped with the cluster showing heightened connectivity with the anterior insula during anticipation as revealed by PPI analyses (shown in yellow). $\boldsymbol{B}$, Scatterplot of the bias index and parameter estimates of the MCC in the simple regression analysis. Note that on the $x$-axis, 1 indicates an equal amount of decisions for pain in the high threat and low threat condition (i.e., no bias).

for the perception of a stimulus as painful, replicating a key finding of our previous study using a similar experimental design (Ploner et al., 2010). The higher the signal level in the anterior insula before stimulus application, the more likely the subsequently applied stimulus was perceived as painful. The results of the present study extend the previous findings by demonstrating that changes in the prestimulus anterior insula activation reflect the integration of information about the salience of the impending stimulation. Activation in this region was lowest before the application of less threatening stimuli which were perceived as nonpainful and highest before more threatening stimuli which were felt as painful (Fig. 3). Note that high and low threat trials were presented in a randomized fashion within each of the four runs, allowing us to test for trial-by-trial changes.

Anatomically, the anterior insula is well suited to integrate different types of information into sensory processing as it is strongly interconnected with other brain regions including the frontal, parietal, and temporal lobe (Mesulam and Mufson, 1982a,b; Mufson and Mesulam, 1982) as well as the cingulate gyrus (Augustine, 1996). In particular, the anterior insula has access to information about emotional aspects of an experience as shown by a large number of studies linking this brain structure with anxiety (for review, see Paulus and Stein, 2006). Findings from these and related studies suggest that the anterior insula plays a key role in interoception which involves the monitoring of sensations that are important for the integrity of the internal body state including the arousal level (Gray et al., 2007) and connecting to systems that are important for allocating attention, evaluating context and planning actions (Craig, 2003; Critchley et al., 2004; Veldhuizen et al., 2007; Singer et al., 2009). Interestingly, activation in the anterior insula has recently been shown to scale with magnitude ratings of pain and visual stimuli (Baliki et al., 2009) providing further evidence for the notion that activation in this region reflects the subjective experience of sensory input rather than their objective features as first reported by Craig et al. (2000). In line with our findings, Lovero et al. (2009) have recently demonstrated that the prestimulus activation in the anterior insula predicts the experience of touch.

To investigate the neural mechanisms by which the anterior insula influences pain perception, we tested for brain regions that showed changes in coupling with the anterior insula when the stimulation was expected to be painful. As shown in Figure 4, the analysis revealed an enhanced prestimulus coupling of the left anterior insula with the anterior part of the MCC as a function of pain anticipation. Anterior insula and MCC are commonly coactivated, particularly in studies involving emotional processing (Craig, 2009). The part of the MCC found here is involved in a number of processes including pain and emotional processing, but also salience, attention to behaviorally relevant stimuli, anticipation, decision-making, motor activity, error detection and conflict (Beckmann et al., 2009). In the context of pain, the MCC has mostly commonly been associated with cognitive aspects and particular attentional processing as opposed to more sensory or affective aspects (for review, see Vogt, 2005). In line with results presented here, Brown and Jones (2008) suggest that this MCCrelated cognitive processing of pain might affect pain processing already during the anticipation period as MCC activation during pain anticipation predicted the degree to which pain interfered with performance of a cognitive task during the application of noxious stimuli. Interestingly, Eippert et al. (2008) recently demonstrated reduced habituation to painful stimuli in the MCC after blocking opioid receptors using naloxone in a conditioned fear paradigm. Together with previous observations on opioid-mediated analgesia in the context of fear conditioning in animals (Fanselow, 1998), this finding suggests that a suppression of the opioid system might underlie a fear-related increase in pain perception.

It has recently been postulated that the anterior insula and the MCC are key nodes of a so-called salience network which detects salient environmental changes regardless of the stimulus modality (Seeley et al., 2007; Taylor et al., 2009). Both studies found a strong temporally correlated low-frequency $(<0.1 \mathrm{~Hz})$ activity during rest between the anterior insula and a region in the cingulate cortex covering the anterior part of the midcingulate cortex extending into the posterior division of the anterior cingulate cortex-a region similar to the one identified in our PPI analysis. Data from tracing studies in monkeys furthermore suggest direct anatomical connections between the anterior insula and the MCC (Mesulam and Mufson, 1982b; Vogt and Pandya, 1987). Based on these findings it seems reasonable to assume that anterior insula and MCC jointly encode the perceived threat value or salience of the upcoming laser stimulation before stimulus encounter. This conclusion is supported by a recent study showing similar anticipatory enhancement of cingulate and insular cortex activation and functional connectivity that is correlated with overestimation of the frequency of aversive pictures following an 
uncertain cue, known as covariation bias (Sarinopoulos et al., 2010).

Because the decision whether a stimulus is perceived as painful is only made after stimulus application, we investigated the stimulus processing in high and low threat trials in more detail. Although all 16 participants showed a threat-related bias toward pain, the bias was stronger in some participants than in others. We therefore tested in which brain areas the activation was positively correlated with the bias toward pain. As shown in Figure 5, the activation in the MCC was most strongly correlated with the bias index: The more often participants rated the stimulation at high-threat sites as painful (compared with stimulation at lowthreat sites), the more active the MCC during stimulation at high threat relative to low threat sites. A subsequent ROI analysis revealed that the MCC activation found in this analysis was located in the cluster showing enhanced coupling with the anterior insula during anticipation. In contrast, activation in the anterior insula during stimulation did not scale with the bias index. Finally, we tested whether the prestimulus coupling between anterior insula and MCC can predict the degree of threat-related bias during stimulation. The threat-related bias was linked to increased functional connectivity between the left anterior insula and the MCC, although the MCC activation found in this analysis was located slightly more lateral than the cluster identified in the initial connectivity analysis and the MCC cluster engaged during stimulation. Based on these data, we propose that the perceived threat-value of a stimulation biases perceptual decision-making in a stepwise fashion. Available information about the threatvalue of an impending stimulation are first processed and integrated in the anterior insula. Depending on the threat-level, the anterior insula then connects with the MCC already before stimulus encounter to tune its sensitivity for the upcoming stimulation. During subsequent stimulus application, the threat-dependent MCC engagement then ensures that potentially more threatening stimuli receive more attention to ensure that they are reliably detected-a mechanism that is often beneficial but might be maladaptive when nonthreatening stimuli are inappropriately treated as harmful.

To conclude, we provide evidence that the anterior insula plays a pivotal role in integrating information about the salience of an impending stimulus into stimulus processing by connecting to the midcingulate cortex. A transition in the salience of pain from pain as a sign of external threat into an internalized disease state has recently been discussed as a key process in the transition from acute to chronic pain (Apkarian et al., 2009). Future investigations of the integrative function of the anterior insula and its underlying neurochemistry might therefore provide a promising target for improving our understanding of pain chronification.

\section{References}

Andersson JL, Hutton C, Ashburner J, Turner R, Friston K (2001) Modeling geometric deformations in EPI time series. Neuroimage 13:903-919.

Apkarian AV, Baliki MN, Geha PY (2009) Towards a theory of chronic pain. Prog Neurobiol 87:81-97.

Arieli A, Sterkin A, Grinvald A, Aertsen A (1996) Dynamics of ongoing activity: explanation of the large variability in evoked cortical responses. Science 273:1868-1871.

Arntz A, Claassens L (2004) The meaning of pain influences its experienced intensity. Pain 109:20-25.

Asmundson GJ, Katz J (2009) Understanding the co-occurrence of anxiety disorders and chronic pain: state-of-the-art. Depress Anxiety 26:888-901.

Augustine JR (1996) Circuitry and functional aspects of the insular lobe in primates including humans. Brain Res Brain Res Rev 22:229-244.

Baliki MN, Geha PY, Apkarian AV (2009) Parsing pain perception between nociceptive representation and magnitude estimation. J Neurophysiol 101:875-887.

Beckmann M, Johansen-Berg H, Rushworth MF (2009) Connectivity-based parcellation of human cingulate cortex and its relation to functional specialization. J Neurosci 29:1175-1190.

Binder JR, Liebenthal E, Possing ET, Medler DA, Ward BD (2004) Neural correlates of sensory and decision processes in auditory object identification. Nat Neurosci 7:295-301.

Boly M, Balteau E, Schnakers C, Degueldre C, Moonen G, Luxen A, Phillips C, Peigneux P, Maquet P, Laureys S (2007) Baseline brain activity fluctuations predict somatosensory perception in humans. Proc Natl Acad Sci U S A 104:12187-12192.

Brown CA, Jones AK (2008) A role for midcingulate cortex in the interruptive effects of pain anticipation on attention. Clin Neurophysiol 119: $2370-2379$.

Craig AD (2003) Interoception: the sense of the physiological condition of the body. Curr Opin Neurobiol 13:500-505.

Craig AD (2009) How do you feel—now? The anterior insula and human awareness. Nat Rev Neurosci 10:59-70.

Craig AD, Chen K, Bandy D, Reiman EM (2000) Thermosensory activation of insular cortex. Nat Neurosci 3:184-190.

Critchley HD, Wiens S, Rotshtein P, Ohman A, Dolan RJ (2004) Neural systems supporting interoceptive awareness. Nat Neurosci 7:189-195.

de Gier M, Peters ML, Vlaeyen JW (2003) Fear of pain, physical performance, and attentional processes in patients with fibromyalgia. Pain 104:121-130.

de Lafuente V, Romo R (2005) Neuronal correlates of subjective sensory experience. Nat Neurosci 8:1698-1703.

Eccleston C, Crombez G (1999) Pain demands attention: a cognitiveaffective model of the interruptive function of pain. Psychol Bull 125:356-366

Eippert F, Bingel U, Schoell E, Yacubian J, Büchel C (2008) Blockade of endogenous opioid transmission enhances acquisition of conditioned fear in humans. J Neurosci 28:5465-5472.

Fanselow MS (1998) Pavlovian conditioning, negative feedback, and blocking: mechanisms that regulate association formation. Neuron 20:625-627.

Friston KJ, Ashburner J, Frith CD, Poline JB, Heather JD, Frackowiak RS (1995) Spatial registration and normalization of images. Hum Brain Mapp 3:165-189.

Friston KJ, Buechel C, Fink GR, Morris J, Rolls E, Dolan RJ (1997) Psychophysiological and modulatory interactions in neuroimaging. Neuroimage 6:218-229.

Gray MA, Harrison NA, Wiens S, Critchley HD (2007) Modulation of emotional appraisal by false physiological feedback during fMRI. PLOS One 2:e546.

Heekeren HR, Marrett S, Bandettini PA, Ungerleider LG (2004) A general mechanism for perceptual decision-making in the human brain. Nature 431:859-862.

Hesselmann G, Kell CA, Eger E, Kleinschmidt A (2008) Spontaneous local variations in ongoing neural activity bias perceptual decisions. Proc Natl Acad Sci U S A 105:10984-10989.

Jackson T, Pope L, Nagasaka T, Fritch A, Iezzi T, Chen H (2005) The impact of threatening information about pain on coping and pain tolerance. $\mathrm{Br} \mathrm{J}$ Health Psychol 10:441-451.

Kaiser J, Lennert T, Lutzenberger W (2007) Dynamics of oscillatory activity during auditory decision making. Cereb Cortex 17:2258-2267.

Lovero KL, Simmons AN, Aron JL, Paulus MP (2009) Anterior insular cortex anticipates impending stimulus significance. Neuroimage 45:976-983.

Mesulam MM, Mufson EJ (1982a) Insula of the old world monkey. I. Architectonics in the insulo-orbito-temporal component of the paralimbic brain. J Comp Neurol 212:1-22.

Mesulam MM, Mufson EJ (1982b) Insula of the old world monkey. III. Efferent cortical output and comments on function. J Comp Neurol 212:38-52.

Moseley GL, Arntz A (2007) The context of a noxious stimulus affects the pain it evokes. Pain 133:64-71.

Mufson EJ, Mesulam MM (1982) Insula of the old world monkey. II. Afferent cortical input and comments on the claustrum. J Comp Neurol 212:23-37.

Paine P, Kishor J, Worthen SF, Gregory LJ, Aziz Q (2009) Exploring rela- 
tionships for visceral and somatic pain with autonomic control and personality. Pain 144:236-244.

Paulus MP, Stein MB (2006) An insular view of anxiety. Biol Psychiatry 60:383-387.

Pleger B, Ruff CC, Blankenburg F, Bestmann S, Wiech K, Stephan KE, Capilla A, Friston KJ, Dolan RJ (2006) Neural coding of tactile decisions in the human prefrontal cortex. J Neurosci 26:12596-12601.

Ploner M, Lee MC, Wiech K, Bingel U, Tracey I (2010) Prestimulus functional connectivity determines pain perception in humans. Proc Natl Acad Sci U S A 107:355-360.

Preuschhof C, Heekeren HR, Taskin B, Schubert T, Villringer A (2006) Neural correlates of vibrotactile working memory in the human brain. J Neurosci 26:13231-13239.

Sarinopoulos I, Grupe DW, Mackiewicz KL, Herrington JD, Lor M, Steege EE, Nitschke JB (2010) Uncertainty during anticipation modulates neural responses to aversion in human insula and amygdala. Cereb Cortex 20:929-940.

Sawamoto N, Honda M, Okada T, Hanakawa T, Kanda M, Fukuyama H, Konishi J, Shibasaki H (2000) Expectation of pain enhances responses to nonpainful somatosensory stimulation in the anterior cingulate cortex and parietal operculum/posterior insula: an event-related functional magnetic resonance imaging study. J Neurosci 20:7438-7445.

Seeley WW, Menon V, Schatzberg AF, Keller J, Glover GH, Kenna H, Reiss AL, Greicius MD (2007) Dissociable intrinsic connectivity networks for salience processing and executive control. J Neurosci 27:2349-2356.

Severeijns R, van den Hout MA, Vlaeyen JW, Picavet HS (2002) Pain cata- strophizing and general health status in a large Dutch community sample. Pain 99:367-376.

Singer T, Critchley HD, Preuschoff K (2009) A common role of insula in feelings, empathy and uncertainty. Trends Cogn Sci 13:334-340.

Smith WB, Gracely RH, Safer MA (1998) The meaning of pain: cancer patients' rating and recall of pain intensity and affect. Pain 78:123-129.

Supèr H, van der Togt C, Spekreijse H, Lamme VA (2003) Internal state of monkey primary visual cortex (V1) predicts figure-ground perception. J Neurosci 23:3407-3414.

Taylor KS, Seminowicz DA, Davis KD (2009) Two systems of resting state connectivity between the insula and cingulate cortex. Hum Brain Mapp 30:2731-2745.

Veldhuizen MG, Bender G, Constable RT, Small DM (2007) Trying to detect taste in a tasteless solution: Modulation of early gustatory cortex by attention to taste. Chem Senses 32:569-581.

Vlaeyen JW, Hanssen M, Goubert L, Vervoort T, Peters M, van Breukelen G, Sullivan MJ, Morley S (2009) Threat of pain influences social context effects on verbal pain report and facial expression. Behav Res Ther 47:774-782.

Vogt BA (2005) Pain and emotion interactions in subregions of the cingulate gyrus. Nat Rev Neurosci 6:533-544.

Vogt BA, Pandya DN (1987) Cingulate cortex of the rhesus monkey: II. Cortical afferents. J Comp Neurol 262:271-289.

Warnaby CE (2005) Thulium fibre laser nerve stimulation and its application in human pain research. $\mathrm{PhD}$ thesis, University of Manchester. 